\title{
Accounting for Beneficial Effects of Worked Examples in Tutored Problem Solving
}

\author{
Ron J. C. M. Salden • Kenneth R. Koedinger • \\ Alexander Renkl • Vincent Aleven • Bruce M. McLaren
}

(C) Springer Science+Business Media, LLC 2010

\begin{abstract}
Recent studies have tested the addition of worked examples to tutored problem solving, a more effective instructional approach than the untutored problem solving used in prior worked example research. These studies involved Cognitive Tutors, software designed to support problem solving while minimizing extraneous cognitive load by providing prompts for problem sub-goals, step-based immediate feedback, and context-sensitive hints. Results across eight studies in three different domains indicate that adding examples to Cognitive Tutors is beneficial, particularly for decreasing the instructional time needed and perhaps also for achieving more robust learning outcomes. These studies bolster the practical importance of examples in learning, but are also of theoretical interest. By using a stronger control condition than previous studies, these studies provide a basis for refining Cognitive Load Theory explanations of the benefits of examples. Perhaps, in addition to other reasons, examples may help simply because they more quickly provide novices with information needed to induce generalized knowledge.
\end{abstract}

Keywords Worked examples · Cognitive tutors · Cognitive load theory

In recent years, a considerable number of studies have explored the conditions under which examples aid in acquiring cognitive skills (for a review, see Atkinson et al. 2000; Renkl 2005, 2010). Among other things, researchers have discovered (1) that examples are more effective than problems in the early stages of skill acquisition, (2) that problems are more effective in later stages (e.g., Kalyuga et al. 2003), (3) that interleaving examples and problems (i.e., example-problem pairs) is more effective than presenting examples and problems in blocks (Trafton and Reiser 1993), (4) that a gradual step-by-step fading of

R. J. C. M. Salden $(\bowtie)$

Department of Computer Science, Worcester Polytechnic Institute, 100 Institute Road, Worcester, MA 01609, USA

e-mail: rsalden@wpi.edu

K. R. Koedinger • V. Aleven • B. M. McLaren

Human-Computer Interaction Institute, Carnegie Mellon University, 5000 Forbes Ave, Pittsburgh, PA 15213, USA

A. Renkl

Psychological Institute, University of Freiburg, Engelbergerstr. 41, D-79085 Freiburg, Germany 
examples to problems is even more effective than example-problem pairs (e.g., Renkl and Atkinson 2003), (5) that examples that make sub-goals explicit are more effective than those that do not (Catrambone 1998), and (6) that the potential of example-based learning is only fully exploited when learners explain the example solutions to themselves (Chi et al. 1989; Renkl 1997).

While the impressive body of research on worked examples to date has been quite successful, it also has two important shortcomings. Firstly, the studies are mostly conducted in a laboratory setting without being extended to the more challenging authentic classroom setting, although there are notable exceptions, including Ward and Sweller's geometry classroom studies (1990). Secondly, the studies have almost exclusively compared learning by studying examples to untutored problem solving. In these studies (e.g., Mwangi and Sweller 1998; Paas 1992; Renkl and Atkinson 2003; Sweller and Cooper 1985; Trafton and Reiser 1993; Van Merriënboer et al. 2002), students in the control condition are sometimes given solutions as feedback after they have attempted a problem, but may not get any feedback at all.

Recently, researchers have started to evaluate the worked example effect in a context in which learners have more instructional support during problem solving. One very successful tutored problem-solving approach is the use of Cognitive Tutors (Koedinger and Aleven 2007). These computer-based tutors provide individualized support for learning by doing (i.e., solving problems) by selecting appropriate problems to be solved, by following alternative student solution strategies, by providing step-by-step feedback, and by making context-sensitive hints and examples of next steps available as needed. This type of tutored problem solving, with step-based immediate feedback, has been shown to be more effective than untutored problem solving, even with whole-solution feedback (e.g., Corbett and Anderson 1995).

Cognitive Tutors individualize the instruction by adapting to alternative solution paths through a process called "model tracing," and by selecting problems based on a model of the students' present knowledge state that is constantly updated through a Bayesian process called "knowledge tracing" (Corbett and Anderson 1995). Adding self-explanation prompts to a Cognitive Tutor has been shown to increase the learners' understanding of domain principles and yield more flexible transfer of knowledge to novel problems (Aleven and Koedinger 2002; see also Roy and Chi 2005; VanLehn et al. 2005).

Cognitive Tutors are being used in over 2,700 US school districts and many full-year classroom evaluations show improved mathematics competence (Koedinger and Aleven 2007) due to Cognitive Tutor courses, as compared with comparison curricula. Through the infrastructure support of the Pittsburgh Science of Learning Center (PSLC; see www. learnlab.org) numerous researchers have been able to conduct studies across multiple domains in both laboratory and classroom-based "in vivo" experiments (cf., Koedinger et al. 2009). A number of such studies have explored the addition of worked examples to Cognitive Tutors. It should be noted that in contrast to most classroom studies using worked examples, PSLC in vivo experiments are part of the actual curricula of the participating high schools and colleges.

Because a tutored environment such as a Cognitive Tutor offers a significant amount of guidance it is a harder control condition then traditional problem solving against which to measure the possible beneficial effects of worked examples. Furthermore, the Cognitive Tutor software environment has several elements that reduce cognitive load during problem solving. These elements include (1) the prompting of sub-goals, (2) immediate feedback on each step a student takes in solving a problem, and (3) the as-needed provision of "bottom-out" hints, that is, worked-out solutions of just those steps students do not generate on their own. 
These instructional elements and their instantiation in individual Cognitive Tutors were originally "guided by eight principles loosely based on the ACT theory" (Anderson et al. 1995). One of these principles, the prompting of sub-goals, was also inspired by early work on Cognitive Load Theory (CLT) (Sweller 1988) and by Catrambone (1998). The commonalities between ACT/ACT-R theory and CLT are strong. Another of those eight Cognitive Tutor principles was to "minimize working memory load" and a relevant tenet of the ACT theory is that learning of skills occurs through a process that compiles the results of interpretative procedures, like reasoning by analogy to worked examples. The result is production rules, which represent knowledge at a finer grain (at a step level) than the problem-level schemas used in theoretical discussions of most worked example studies.

In the current paper, we describe Cognitive Tutors in detail and discuss how they affect relevant cognitive processes for learning in terms of CLT, followed by a review of eight studies that have compared a standard Cognitive Tutors with an example-enriched Cognitive Tutor. Overall, these experiments support the practical importance of examples in learning by showing that, across a number of different implementations, worked examples integrated into an environment that supports tutored problem solving leads to more efficient learning and sometimes to better robust learning outcomes. Finally, we reflect on what the new results might mean at a theoretical level. Can these results be explained by CLT? Might they suggest new ways to refine the theory, particularly with respect to circumstances and mechanisms under which the use of worked examples may make learning more efficient or more effective?

\section{Tutored Problem Solving in Cognitive Tutors}

In a recent review of research on the effectiveness of instructional features of Cognitive Tutors, Koedinger and Aleven (2007) highlighted an "assistance dilemma" between research-based recommendations for more guidance/assistance (Kirschner et al. 2006), on one hand, versus more desirable difficulties (Bjork 1994), on the other hand. Inspired in part by this dilemma, several recent studies have embedded worked examples in a variety of Cognitive Tutors and investigated whether the examples still had beneficial effects over the tougher tutored control condition (e.g., Anthony 2008; McLaren et al. 2008; Salden et al. 2010; Schwonke et al. 2009).

We focus on the Cognitive Tutor used in the studies by Schwonke et al. to illustrate how its features may reduce extraneous cognitive load by limiting the part of the solution space in which students have to search for the next sub-goal to be solved and associated moves for solving it. These features include (a) an interactive, integrated diagram (Butcher and Aleven 2007, 2008), (b) a table which provides all steps or sub-goals, and (c) feedback per step. Other features, like (d) hints only on request and (e) prompts for self-explanation, may increase the effort required but also enhance learning, that is, they may increase "germane cognitive load" (Sweller 2010; Sweller et al. 1998).

Figure 1 shows the interface of the Geometry Cognitive Tutor as used in the tutored problem solving control condition. The students first read the problem statement in the upper left corner where some quantities are given in the text. They can click on the question marks in the integrated diagram which opens a work area (the smaller of the two boxes just to the left of the diagram in Fig. 1) where they have to fill in a numeric value and the name of a corresponding geometric theorem. Once the students fill in the correct value, the question mark is replaced with the numeric value in the diagram. Furthermore, all correct values and corresponding theorems are displayed in the table in the upper right corner, 


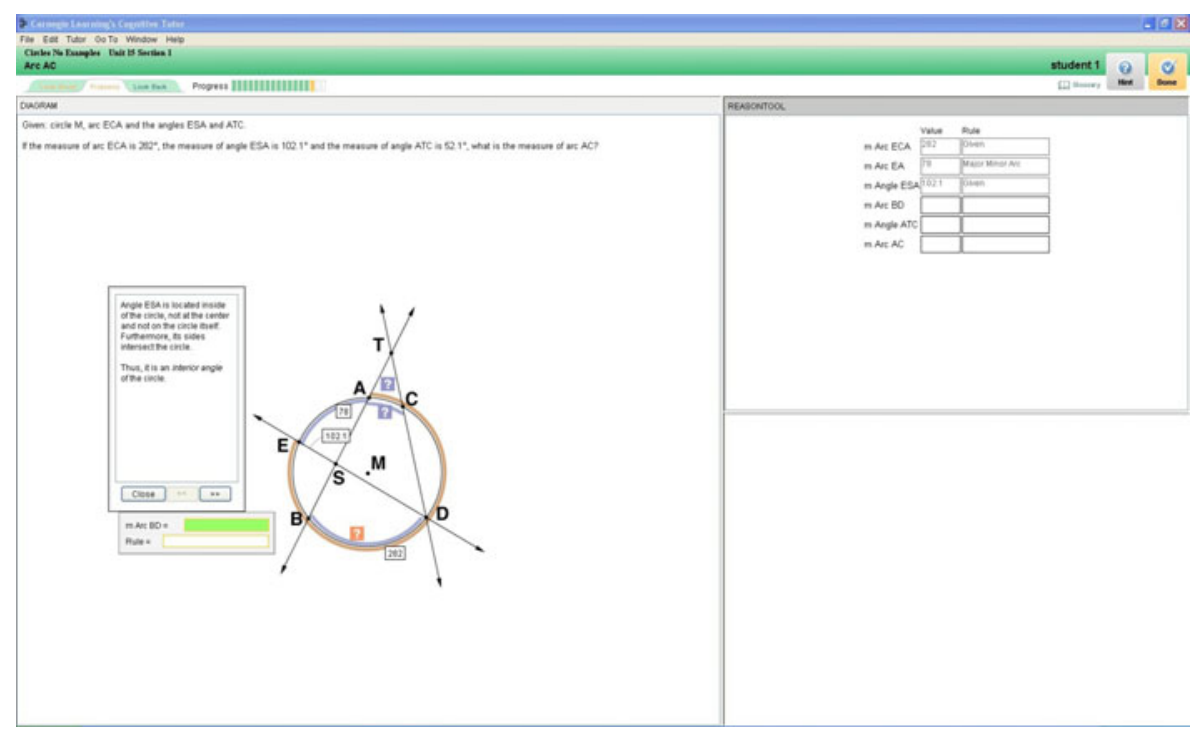

Fig. 1 Screenshot of a student working with a three-step problem in the Standard Geometry Cognitive Tutor

which thus provides a quick and continuously updated overview of the students' progress through the problem at hand.

From the start of each problem, the table lists the steps in order (i.e., in an order that observes the logical dependencies among the steps and in which the problem could be solved). In other words, the table prompts students with the sub-goals that need to be pursued so as to eliminate the need for problem search. It thereby puts the instructional focus on learning the target geometric theorems and associated concepts and skills. Note that although the table with the sub-goals provides some guidance, the steps still need to be completed by the students themselves, which is not the case in worked examples. As such, the table eliminates problem search, that is, the search for which sub-goal to pursue next, but does not eliminate knowledge search, that is, the search for which operator to use to perform a given sub-goal (cf., Aleven and Koedinger 2002).

The Cognitive Tutor provides corrective feedback when a student makes an incorrect entry, whether a numeric value, expression, or theorem. It also gives an error message if students try to work on steps out of the correct order. Additionally, if a student makes too many incorrect attempts on a given sub-goal, the Cognitive Tutor will suggest that the student uses the hints. Each sub-goal has its own sequence of hint levels that end with a "bottom-out hint," which gives a correct entry or answer for that sub-goal. Such bottom-out hints can be thought of as converting a step in a problem from an opportunity to learn by doing into an opportunity to learn by studying an example (the answers given by such hints are the same as the ones provided to students in the example condition described below).

Asking students to provide, for each step, the name of the theorem that justifies the value (see the table in Fig. 1, top right) has been shown to be an effective prompt to engage students in self-explanation and thus enhance their learning of problem solving with understanding (Aleven and Koedinger 2002). The use of self-explanation prompts has been found to improve learning outcomes in a variety of other studies involving Cognitive Tutors (e.g., Corbett et al. 2003; Renkl and Atkinson 2007), in addition to studies without such software (e.g., Chi et al. 1994; Renkl et al. 1998). 
In addition to the above features, the problem set for the studies by Schwonke et al. (2009) was designed to start with easier problems with low intrinsic load (e.g., only one step, see Fig. 2) and then introduce harder problems with higher intrinsic load (e.g., three steps, see Fig. 1). Intrinsic load results from the inherent difficulty of problems and tends to increase as the number of required steps increases. A total of seven problems addressed three geometry theorems, major minor arc, interior angle, and exterior angle. The first three problems addressed each theorem in isolation (i.e., one-step problems); the remaining four problems combined all three theorems (i.e., three-step problems: see Table 1).

Throughout the seven problems, the intrinsic complexity is further increased by addressing different possible ways of applying each theorem and by making the theorems interdependent and thereby increasing element interactivity (Sweller 2010). For example, the major minor arc theorem can be applied using the minor arc to find the major arc or vice versa. To illustrate interdependency of theorems, a major arc or a minor arc might be associated with an interior or exterior angle, or possibly even both arcs might be so associated. In short, by starting off with relatively low intrinsic load, possible overload is avoided. Since the intrinsic complexity of the problems is increased gradually, students are more likely to spend their working memory resources on making inferences that are germane for learning.

\section{Recent Research on Worked Examples in Tutored Problem Solving}

Recently, eight studies have compared a regular Cognitive Tutor with an example-enriched Tutor in the domains of geometry (Salden et al. 2010; Schwonke et al. 2009), chemistry (McLaren et al. 2008), and algebra (Anthony 2008). These studies tested the hypothesis that replacing a substantial number of tutored problems with worked examples would further enhance student learning by reducing instructional time and/or increasing student outcomes in terms of retention and transfer performance.

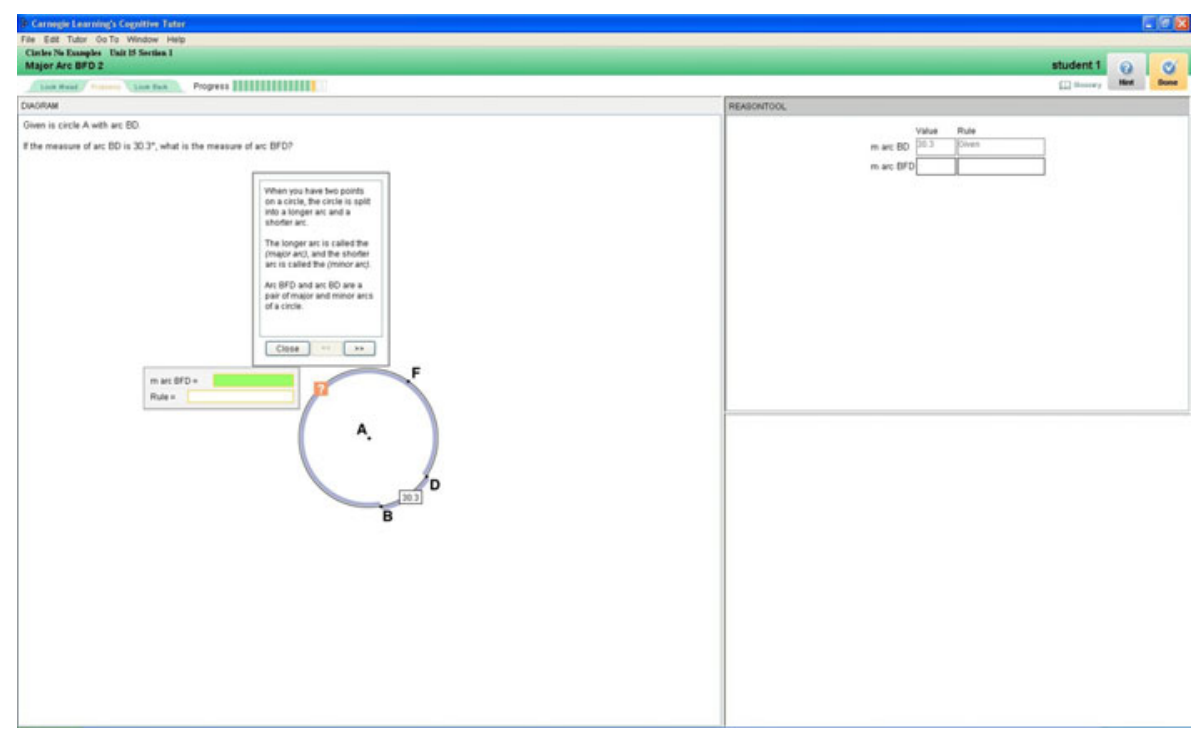

Fig. 2 Screenshot of a student working with a one-step problem in the Standard Geometry Cognitive Tutor 
Table 1 The fading of worked-out steps in the "fixed fading" condition; in problems P1 to P7, steps involving theorems T1 to T3 were worked out (W) initially, and were systematically faded later (S for solving)

\begin{tabular}{|c|c|c|c|c|c|c|}
\hline & \multicolumn{3}{|c|}{ Problem solving } & \multicolumn{3}{|c|}{ Examples } \\
\hline & $\mathrm{T} 1$ & $\mathrm{~T} 2$ & $\mathrm{~T} 3$ & $\mathrm{~T} 1$ & $\mathrm{~T} 2$ & $\mathrm{~T} 3$ \\
\hline P1 & S & & & W & & \\
\hline P2 & & $\mathrm{S}$ & & & W & \\
\hline P3 & & & S & & & W \\
\hline P4 & S & S & S & W & $\mathrm{W}$ & W \\
\hline P5 & S & S & S & W & W & S \\
\hline P6 & S & $\mathrm{S}$ & S & W & $\mathrm{S}$ & $\mathrm{S}$ \\
\hline P7 & $\mathrm{S}$ & S & $\mathrm{S}$ & $\mathrm{S}$ & $\mathrm{S}$ & $\mathrm{S}$ \\
\hline
\end{tabular}

In the example-enriched Geometry Cognitive Tutor of the aforementioned studies (Schwonke et al. (2009), selected numeric answer steps are filled in by the tutor, and a brief explanation of the arithmetic involved in the step (but not the geometric reasoning) is provided (see Fig. 3, smallest of the two boxes just to the left of the diagram). The student's learning goal is to study the worked-out answer step and engage in self-explanation of the geometric reasoning involved in the step, prompted by the requirement that they enter the geometry theorem which explains why the given numeric answer is justified. The interaction is different from the standard Cognitive Tutor where the student has to find the numeric answer step either through problem solving or by requesting hint messages. Note that both standard and example-enriched tutor conditions prompt for self-explanation; in both cases the student must enter the correct corresponding geometry theorem.

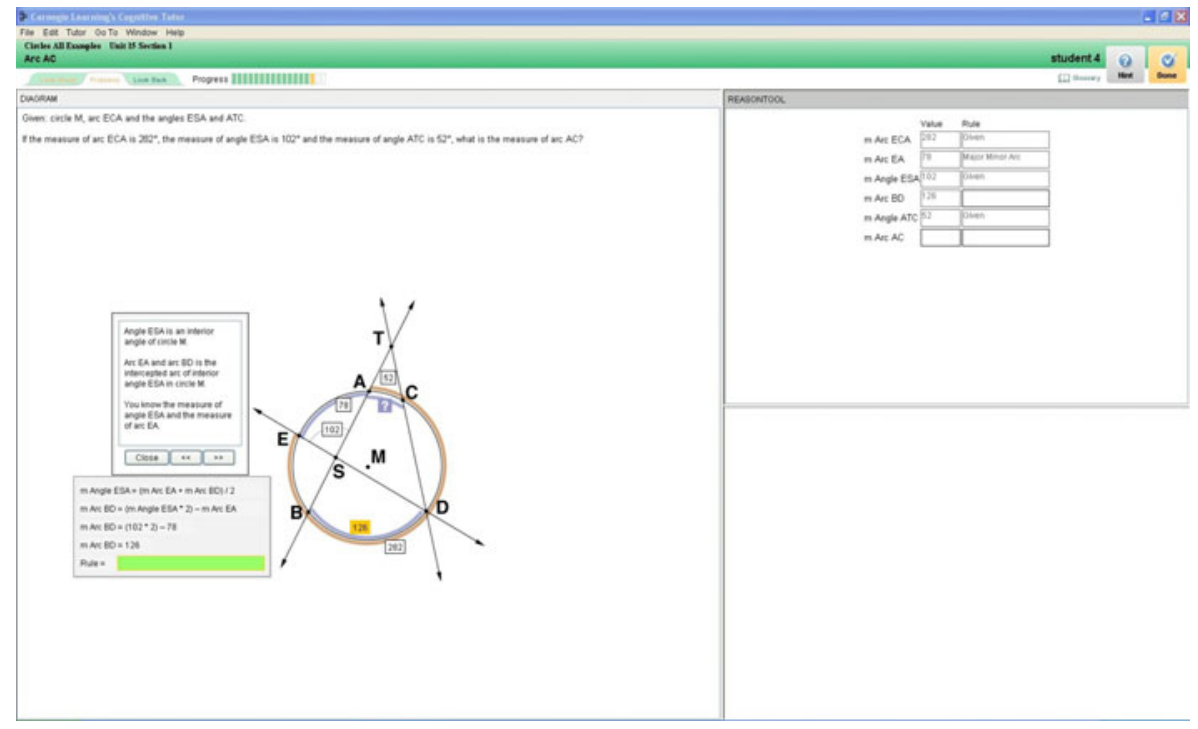

Fig. 3 Screenshot of a student working with a three-step problem in the Example-Enriched Geometry Cognitive Tutor which shows the worked-out solution for the numeric value of arc BD 
The examples in the Schwonke et al. (2009) studies were gradually faded according to a "fixed" fading scheme that was identical for all learners. Students first receive a worked example with all steps worked out. In subsequent problems, increasingly more steps are not worked out but are left open for the learner to complete, with the guidance of the tutor. Thus, these partially faded examples are like completion problems (Paas 1992; van Merriënboer et al. 2002), except that tutoring assistance is provided on the open steps. The fading scheme was the same for all learners (see Table 1). Once the examples were fully faded, the students in the example condition received tutored problems just like the students in the control condition (see Fig. 1). The results from both studies indicated that tutored problem solving combined with example fading led to at least as much learning and transfer in significantly less instructional time. The second study also found better transfer to conceptually oriented problems from the example-enriched tutor than from tutored problem solving alone.

Salden et al. (2010) conducted two follow-up studies, one lab study and one classroom study, using the Geometry Cognitive Tutor (Schwonke et al. 2009) in which they compared the same tutored problem-solving and fixed-fading conditions with respect to learning outcomes on procedural and conceptual transfer tests as well as on a long-term retention test. Similar to the first geometry study, no significant differences were found on either transfer test. However, in contrast to the earlier two geometry studies the example fading condition did not need less instructional time.

These two studies also had a third experimental condition where worked example steps were adaptively faded by the software based on how well students had self-explained prior steps in the tutor's problem sequence that involve the same knowledge component. In this adaptive fading procedure, the one-step problems early on in the problem sequence (P1 to P3 in Table 1) were all worked out; the steps in the subsequent three-step problems (P4 to P7 in Table 1) were then faded in an adaptive (i.e., individualized) manner. Both studies revealed evidence of improved learning results on a long-term retention test from adaptive fading over fixed fading and problem solving.

The instructional efficiency findings of the first two geometry studies (Schwonke et al. 2009) were corroborated by McLaren et al. (2008) who conducted three classroom studies in which a Chemistry Cognitive Tutor was compared with an example-enhanced Tutor. The students in the examples conditions received example-problem pairs; for each example they watched a narrated video of a problem being solved in the tutor interface by an expert and, in the two later studies, were prompted for self-explanations of the problem solving performed in the videos. The self-explanation prompts were multiple-choice questions that had to be answered correctly before the student could move on. Students showed significant pre-to-post learning in both conditions in all three studies, but there was no difference between conditions on posttest performance. Students learned just as much from either tutor versions, but importantly, the students using the example-enriched tutor learned significantly more efficiently, using $21 \%$ less time to finish the same set of problems. Unlike the Geometry studies, these studies did not assess conceptual transfer.

Anthony (2008) conducted a classroom study in which the standard Algebra Cognitive Tutor was compared with a version that included example-problem pairs consisting of annotated worked examples presented with problem-solving tasks. Although no significant differences on an immediate retention test were revealed, students who learned with examples attained significantly better long-term retention scores. Additionally, both the examples Tutor condition and the regular Cognitive Tutor condition experienced a similar amount of mental effort. The students' mental effort was measured using a nine-point subjective rating scale (Paas 1992) presented after they finished the training phase. 
It should be noted that in all of these studies about one half of the problem-solving steps in the standard tutors were replaced with examples in the example-enriched tutors. Both the Chemistry and Algebra studies used example-problem pairs in comparison to problemproblem pairs. The earlier two Geometry studies used a fixed fading approach in which (as shown in Table 1) after three one-step examples, half of the steps in the four subsequent three-step problems were worked out. Lastly, in the adaptive fading condition of the later two Geometry studies, the participants also received three one-step examples before the four subsequent three-step problems were adaptively faded based on how well students selfexplained the corresponding numeric steps.

Across these eight studies four different types of post-assessment measures were used: immediate problem-solving test, long-term retention test, procedural and conceptual transfer tests. The example-enriched Tutors showed beneficial learning outcomes for long-term retention in three (Anthony 2008; Salden et al. 2010) out of three studies, with five studies not including long-term retention. The example-enriched Tutors showed benefits for conceptual transfer in one (Schwonke et al. 2009) out of four studies with four studies not including conceptual transfer. For five of these eight studies, the exampleenriched Tutors led to a more efficient use of instructional time where students obtained similar learning outcomes as students in the standard Cognitive Tutors while spending significantly less time. In none of the studies did inclusion of examples increase instructional time or reduce learning on any outcome measure.

Overall, these findings seem to indicate that adding worked examples to tutored problem solving may enhance learning, but not as much as adding examples to untutored problem solving where most studies have shown better learning outcomes than when novice students work on untutored problems alone (e.g., Mwangi and Sweller 1998; Paas 1992; Sweller and Cooper 1985; Trafton and Reiser 1993; van Merriënboer et al. 2002; Ward and Sweller 1990). This result is consistent with the notion that tutored problem solving poses a more challenging control condition than the untutored problem solving condition used in most prior worked example studies. Additionally, similar to previous beneficial findings of worked examples in untutored problem solving, the addition of examples appears to reduce the instructional time needed.

\section{Cognitive Load Theory Explanation of the Worked Example Effect}

As a first step in considering the theoretical significance of these results, we start by briefly summarizing the traditional CLT explanation for the worked examples effect. In the next section, we consider how the results about worked examples in tutored problem solving can be used to reflect upon and refine CLT.

CLT argues that worked examples improve learning because they reduce extraneous load produced during problem solving (e.g., Sweller et al. 1998). In early skill acquisition, the novice learner lacks relevant prior knowledge in long-term memory. As a consequence, novice learners must resort to general problem-solving strategies, such as means-ends analysis, which require a substantial portion of working memory capacity. Learners must maintain many items in working memory including the current problem state, the goal state, differences between these states, and problem sub-goals. This demand can lead to cognitive overload.

Although general strategies such as means-ends analysis can be effective problemsolving strategies, they are "exceptionally expensive of working memory capacity" (Sweller 1988), and "bear little relation to schema construction processes that are concerned with learning to recognize problem states and their associated moves" (Sweller et al. 1998, p. 271). 
Accordingly, CLT regards conventional problem solving as an inefficient technique for constructing expert-like schemas because problem-level means-ends analysis requires the storage of sub-goals and places a heavy load on novice learners. This load can be reduced by presenting worked examples or goal-free problems (Sweller et al. 1998), which do not require the cognitive resources needed to search for a particular problem solution. Thus, learners have more resources available to attend to problem states and associated moves.

\section{Unpacking the Process: What Causes Load in Tutored Problem Solving?}

In this section we investigate how the traditional CLT explanation for the benefits of adding worked examples to untutored problem solving may be extended and perhaps refined to explain the benefits of adding worked examples in tutored problem solving. As discussed earlier, Cognitive Tutors reduce extraneous cognitive load by limiting part of the solution space students have to search for sub-goals and associated moves, and increase germane cognitive load by guiding students through the solution space. In fact, the next sub-goal is presented to the student and s/he can actively get an example by asking the tutor to reveal the next move to make by going to the "bottom-out" hint.

Although the next move is not available up front in a tutored problem like it is in an example, sometimes students succeed in generating the next move on their own. They may do so, for instance, (1) by retrieving a previously acquired, but perhaps not fully learned, production rule or schema, (2) by reasoning by analogy to a prior example in memory or in the tutor's glossary, or (3) by interpreting and applying a textbook rule or a tutor hint message (c.f., Anderson et al. 1997).

In such cases of successful self-generation of a correct next move (and without requiring problem-level means-ends search), it is plausible that students learn as much as or more than if they were given an example (cf., Roediger and Karpicke 2006). That is, such selfgeneration of correct responses, like that observed in the expertise reversal effect (Kalyuga et al. 2003), may be more germane than extraneous and may enhance robust learning outcomes. Tutored problem solving is designed to maximize self-generation opportunities and does so in a way that is not possible in the untutored problem-solving control condition in worked example studies.

For novice students, however, many problem-solving situations, even with a Cognitive Tutor, do not end with successful self-generation. Even with immediate feedback on errors and layers of hints, students may need a bottom-out hint in which they are told the next move. This situation is of particular interest theoretically because it is significantly different than in the typical untutored problem-solving control condition. In contrast to untutored problem solving, students working in a Cognitive Tutor have exactly the same information after receiving a bottom-out hint as students in the example condition who were given the next move up front. Furthermore, they are in a similar working memory state. In particular, they avoid the concurrent cognitive load caused by the need to store problem sub-goals while performing means-ends search, which is characteristic of untutored problem solving. Tutored problem solving can eliminate this load by providing the next sub-goal. For instance, the next angle to find is prompted in the table in the Geometry Cognitive Tutor described above. In this situation, just as in the example condition, no concurrent load is required and the student is freed of this potentially extraneous load to focus his attention on the provided next move and on inducing a general schema that explains the example.

Shih et al. (2008) did a careful analysis of student individual differences at this point, right after receiving a bottom-out hint. The findings indicate that some students spend more 
time than others and that the time spent is positively correlated with learning gains. This observation is consistent with the hypothesis (and Chi et al.'s (1989) observation) that some students spontaneously engage in deeper self-explanations of examples than others. However, whereas the students in Chi's study engaged in such activity during example study, the students in Shih's study used the standard Cognitive Tutor's bottom-out hints to produce worked examples. (Recall that students may also be prompted to self-explain in some Cognitive Tutors as was illustrated in Figs. 1, 2, and 3.)

If there is a benefit of worked examples above and beyond eliminating load-inducing storage of sub-goals as done in the standard Cognitive Tutors, how might the extra next-move information in examples be beneficial nevertheless? There are two potential lines of reasoning that are consistent with our results. Firstly, the most consistent finding in our studies is the reduced instructional time required in learning from example-enriched tutors relative to the standard tutors. In standard tutored problem solving, the tutor guarantees that students get to each next move, but whether the student does so through their own reasoning or after some rounds of interaction with the tutor, it takes longer to get there than when it is given in an example. The Tutor timing data supports this point: students spend 21\% (McLaren et al. studies) and 19\% (Schwonke et al. studies) more time working with the standard Cognitive Tutor. Once a student gets to a next move, with or without tutor help, they may learn just as much. In this line of reasoning, the "cognitive load" produced by tutored problem solving is in the extra time required to produce the next move even when that effort does not require problem-level means-ends analysis and problem sub-goal storage.

A second line of reasoning suggests that other factors might be at play in addition to the time difference. The effort required to produce a next move may "spill over" into the phase after the next move has been given (in the case that the student requests a bottom-out hint) such that the student does not study the next move in these circumstances (i.e., when they reach the bottom-out hint after struggling with the step) as much as they do when the example is given up front (see also Schnotz 2010). This "spill over" could be general load/ fatigue like hemoglobin depletion (e.g., Grandjean 1979; Watanabe et al. 2002) or performance orientation (Dweck and Leggett 1988; Elliot and McGregor 2001).

Renkl and colleagues (Hilbert et al. 2008; Renkl and Atkinson 2010) have discussed how performance orientation may be induced by an exclusive focus on problem-solving practice and how a resulting dysfunctional allocation of attentional resources may prevent students from engaging in learning to acquire an understanding of domain principles. Performance-oriented students may employ shallow strategies to guess at numeric answers when confronted with problems. For example, they may use shallow strategies such as keyword strategies (i.e., selecting a procedure by a keyword in the cover story of a problem) or a copyand-adapt strategy (i.e., copying the solution procedure from a presumably similar problem and adapting the numbers in the procedure). While traditional problem-solving instruction usually provides only a single example, more examples should be provided to fully exploit the potential of example-based learning. Such an approach is more likely to develop an attentional focus on how to apply domain principles. Against this background, studying worked example steps in Cognitive Tutors may enhance attention to understanding how principles are to be applied. This initial understanding can be deepened during subsequent problem solving.

\section{Discussion}

We have discussed eight studies showing positive effects of examples in Cognitive Tutors. The findings indicate that adding worked examples to tutored problem solving reduces 
instructional time and may enhance learning outcomes. These results extend similar outcomes from previous work using untutored problem solving to the more challenging control environment of Cognitive Tutors.

The benefits of worked examples in tutored problem solving are not consistent with the idea that the primary effect of examples is due to the fact that they eliminate the need to store sub-goals during means-ends analysis. In fact, tutored problem solving has important similarities with the goal-free strategy of Sweller et al. (1998) and the completion problems of van Merriënboer and Sweller (2005). They are approaches to problem-based instruction that focus students' attention on problem states and useful solution steps to produce better induction of general schemas. In many Cognitive Tutors, as in goal-free problems and completion problems, problem-level means-ends analysis is not required, so adding examples cannot eliminate it. What might be an alternative explanation, then, for the apparent benefits of adding worked examples to tutored problem solving?

A first alternative takes the view that an example-enriched tutor does not change the learning process per se, but simply reduces the instructional time needed. The examples, which are provided for about half of the problem steps, eliminate the need for novices to generate next moves; either on their own or after some interaction with the tutor, and thus students need less time before they can use a next move to induce a schema. For novice learners, the extra time required to struggle on all problem-solving steps (in the standard tutor) rather than about half of them (in example-enhanced tutor) does not seem to reap any measurable learning benefits. Furthermore, this view suggests that once a correct next step is available the learning process is the same whether or not the student struggled to produce it or it was given to them (cf., Anderson et al. 1995). This "time-savings" explanation requires the assumption that the observed positive effects on long-term retention (three of three studies) and conceptual transfer measures (one of four studies) are spurious.

On the other hand, these effects on learning outcome may reflect real differences. In that case, an explanation is needed for why the learning process may be worse after a struggle to produce a next step than when the next step is simply given. It may be that the effort required to produce a next move "spills over" into the next phase (i.e., the phase where, after struggling on the step, the move is available, possibly through the bottom-out hint), causing the student to spend less time on example study than when the example is given up front. This spill over is not the kind of concurrent load that is produced from problem-level means-ends analysis in untutored problem solving situations, but it may be a more global load or fatigue that, though not concurrent, may still inhibit the depth of student processing of an available next move (Schnotz 2010).

One future research goal is to investigate this proposed spill-over mechanism by comparing success rates on the reason steps (e.g., reason for arc BD in Fig. 3) between the standard and example-enhanced tutor conditions. If the load is isolated to producing the next move in the standard Cognitive Tutor, then there would be no effort spill over and the success rates of the corresponding reason steps should not differ significantly. However, if students working in the standard Cognitive Tutor experience effort spill over, preventing them from adequately studying the bottom-out hints, then they can be expected to make more errors on the corresponding reason steps than students in the example-enhanced tutor.

An alternative to global load spill over is the idea that exclusive problem solving may put students in a performance orientation mode where they are trying to complete problems rather than study solutions to prepare for future problems. It may be interesting and productive to investigate changes in motivational factors associated with adding worked examples to tutors, for instance, does doing so reduce "gaming the system" behaviors (Baker et al. 2004)? 
More generally, further studies of the detailed log data produced by student-tutor interactions could be used to better probe when students engage in dysfunctional allocation of attentional resources. Cognitive Tutors log all student actions and this data may enable the identification of shallow strategies such as key word strategies or a copy-and-adapt strategy through student behaviors and error patterns present in the log data (cf., Shih et al. 2008). Additionally, researchers might employ eye tracking to study the allocation of students' attention by measuring the amount of time they spend looking at different parts of the interface in different instructional conditions.

The results of the adaptive fading condition in the two geometry studies by Salden et al. (2010) point to a theoretically important difference between a focus on learning problemlevel schemas in many CLT studies, which does not seem to explain the benefit of steplevel adaptive fading, and a focus on step-level learning of finer-grained knowledge components in tutored problem solving. Lastly, not only do these findings extend our previous work, they also fit rather well with the future direction of CLT as suggested by van Merriënboer and Sweller (2005) in terms of adaptive e-Learning (see also Kalyuga and Sweller 2005). More specifically, the Cognitive Tutors' adaptive nature is highly suited to fulfill the CLT interest in measures that reflect the quality of available cognitive schemata and changes of the expertise level of individual students.

As such, future research should investigate whether this adaptivity can increase the acquisition of flexible problem-solving skills (Kalyuga et al. 2010), which in turn is expected to lead to better preparation for future learning (Bransford and Schwartz 1999; Chi and VanLehn 2007). The students in the adaptive fading of examples condition of Salden et al. (2010) obtained better long-term retention and potentially could reveal more flexible problem-solving skills on long-term transfer tests.

\section{References}

Aleven, V., \& Koedinger, K. R. (2002). An effective meta-cognitive strategy: Learning by doing and explaining with a computer-based cognitive tutor. Cognitive Science, 26, 147-179.

Anderson, J. R., Corbett, A. T., Koedinger, K. R., \& Pelletier, R. (1995). Cognitive tutors: Lessons learned. The Journal of the Learning Sciences, 4, 167-207.

Anderson, J. R., Fincham, J. M., \& Douglass, S. (1997). The role of examples and rules in the acquisition of a cognitive skill. Journal of Experimental Psychology: Learning, Memory, and Cognition, 23, 932-945.

Anthony, L. (2008). Developing handwriting-based Intelligent Tutors to enhance mathematics learning. Unpublished doctoral dissertation, Carnegie Mellon University, USA.

Atkinson, R. K., Derry, S. J., Renkl, A., \& Wortham, D. W. (2000). Learning from examples: Instructional principles from the worked examples research. Review of Educational Research, 70, 181-214.

Baker, R. S., Corbett, A. T., Koedinger, K. R., \& Wagner, A. Z. (2004). Off-task behavior in the Cognitive Tutor classroom: When students "game the system". In: Proceedings of ACM CHI 2004: ComputerHuman Interaction. pp. 383-390.

Bjork, R. A. (1994). Memory and metamemory considerations in the training of human beings. In J. Metcalfe \& A. Shimamura (Eds.), Metacognition: Knowing about knowing (pp. 185-205). Cambridge: MIT.

Bransford, J. D., \& Schwartz, D. L. (1999). Rethinking transfer: A simple proposal with multiple implications. In A. Iran-Nejad \& P. D. Pearson (Eds.), Review of research in education, Vol. 24 (pp. 61100). Washington, DC: American Educational Research Association.

Butcher, K., \& Aleven, V. (2007). Integrating visual and verbal knowledge during classroom learning with computer tutors. In D. S. McNamara \& J. G. Trafton (Eds.), Proceedings of the 29th Annual Conference of the Cognitive Science Society (pp. 137-142). Austin: Cognitive Science Society.

Butcher, K., \& Aleven, V. (2008). Diagram interaction during intelligent tutoring in geometry: Support for knowledge retention and deep transfer. In C. Schunn (Ed.), Proceedings of the 30th Annual Meeting of the Cognitive Science Society, CogSci 2008 (pp. 894-899). Austin: Cognitive Science Society. 
Catrambone, R. (1998). The subgoal learning model: Creating better examples so that students can solve novel problems. Journal of Experimental Psychology: General, 127, 355-376.

Chi, M., \& VanLehn, K. (2007). Accelerated future learning via explicit instruction of a problem solving strategy. In R. Luckin, K. R. Koedinger, \& J. Greer (Eds.), Proceedings of the 13th International Conference on Artificial Intelligence in Education (pp. 409-416). Amsterdam: IOS.

Chi, M. T. H., Bassok, M., Lewis, M. W., Reimann, P., \& Glaser, R. (1989). Self-explanations: How students study and use examples in learning to solve problems. Cognitive Science, 13, 145-182.

Chi, M. T. H., de Leeuw, N., Chiu, M., \& LaVancher, C. (1994). Eliciting self-explanations improves understanding. Cognitive Science, 18, 439-477.

Corbett, A. T., \& Anderson, J. R. (1995). Knowledge tracing: Modeling the acquisition of procedural knowledge. User Modeling and User-Adapted Interaction, 4, 253-278.

Corbett, A. T., Wagner, A., \& Raspat, J. (2003). The Impact of analyzing example solutions on problem solving in a pre-algebra tutor. In: Proceedings of AIED 2003: The 11th International Conference on Artificial Intelligence and Education. pp. 133-140.

Dweck, C. S., \& Leggett, E. L. (1988). A social-cognitive approach to motivation and personality. Psychological Review, 95, 256-273.

Elliot, A. J., \& McGregor, H. A. (2001). A $2 \times 2$ achievement goal framework. Journal of Personality and Social Psychology, 80, 501-519.

Grandjean, E. (1979). Fatigue in industry. British Journal of Industrial Medicine, 36, 175-186.

Hilbert, T. S., Renkl, A., Schworm, S., Kessler, S., \& Reiss, K. (2008). Learning to teach with worked-out examples: A computer-based learning environment for teachers. Journal of Computer-Assisted Learning, 24, 316-332.

Kalyuga, S., \& Sweller, J. (2005). Rapid dynamic assessment of expertise to improve the efficiency of adaptive e-learning. Educational Technology, Research and Development, 53, 83-93.

Kalyuga, S., Ayres, P., Chandler, P., \& Sweller, J. (2003). The expertise reversal effect. Educational Psychologist, 38, 23-32.

Kalyuga, S., Renk1, A., \& Paas, F. (2010). Facilitating flexible problem solving: A cognitive load perspective. Educational Psychology Review, 22, 175-186.

Kirschner, P. A., Sweller, J., \& Clark, R. E. (2006). Why minimal guidance during instruction does not work: An analysis of the failure of constructivist, discovery, problem-based, experiential, and inquiry-based teaching. Educational Psychologist, 41, 75-86.

Koedinger, K. R., \& Aleven, V. (2007). Exploring the assistance dilemma in experiments with cognitive tutors. Educational Psychology Review, 19, 239-264.

Koedinger, K. R., Aleven, V., Roll, I., \& Baker, R. (2009). In vivo experiments on whether supporting metacognition in intelligent tutoring systems yields robust learning. In: D. J. Hacker, J. Dunlosky, \& A. C. Graesser (Eds.) Handbook of metacognition in education (pp. 897-964). The Educational Psychology Series. New York: Routledge.

McLaren, B. M., Lim, S., \& Koedinger, K. R. (2008). When and how often should worked examples be given to students? New results and a summary of the current state of research. In B. C. Love, K. McRae, \& V. M. Sloutsky (Eds.), Proceedings of the 30th Annual Conference of the Cognitive Science Society (pp. 2176-2181). Austin: Cognitive Science Society.

Mwangi, W., \& Sweller, J. (1998). Learning to solve compare word problems: The effect of example format and generating self-explanations. Cognition and Instruction, 16, 173-199.

Paas, F. (1992). Training strategies for attaining transfer of problem-solving skill in statistics: A cognitiveload approach. Journal of Educational Psychology, 84, 429-434.

Renkl, A. (1997). Learning from worked-out examples: A study on individual differences. Cognitive Science, $21,1-29$.

Renkl, A. (2005). The worked-out-example principle in multimedia learning. In R. Mayer (Ed.), Cambridge handbook of multimedia learning. Cambridge: Cambridge University Press.

Renkl, A. (2010). Instruction based on examples. In R. E. Mayer \& P. A. Alexander (Eds.), Handbook of research on learning and instruction. New York: Routledge.

Renkl, A., \& Atkinson, R. K. (2003). Structuring the transition from example study to problem solving in cognitive skills acquisition: A cognitive load perspective. Educational Psychologist, 38, 15-22.

Renkl, A., \& Atkinson, R. K. (2007). Cognitive skill acquisition: Ordering instructional events in examplebased learning. In F. E. Ritter, J. Nerb, E. Lehtinen, \& T. O'Shea (Eds.), In order to learn: How ordering effect in machine learning illuminate human learning and vice versa. Oxford: Oxford University Press.

Renkl, A., \& Atkinson, R. K. (2010). Learning from worked-out examples and problem solving. In J. Plass, R. Moreno, \& R. Brünken (Eds.), Cognitive load theory and research in educational psychology (pp. 91-108). New York: Cambridge University Press. 
Renkl, A., Stark, R., Gruber, H., \& Mandl, H. (1998). Learning from worked-out examples: The effects of example variability and elicited self-explanations. Contemporary Educational Psychology, 23, 90-108.

Roediger, H. L., \& Karpicke, J. D. (2006). The power of testing memory: Basic research and implications for educational practice. Perspectives on Psychological Science, 1, 181-210.

Roy, M., \& Chi, M. T. H. (2005). Self-explanation in a multi-media context. In R. Mayer (Ed.), Cambridge handbook of multimedia learning (pp. 271-286). Cambridge, UK: Cambridge Press.

Salden, R. J. C. M., Aleven, V., Schwonke, R., \& Renkl, A. (2010). The expertise reversal effect and worked examples in tutored problem solving. Instructional Science, 38, 289-307.

Schnotz, W. (2010). Reanalyzing the expertise-reversal effect. Educational Psychology Review, 38, $315-323$.

Schwonke, R., Renkl, A., Krieg, C., Wittwer, J., Aleven, V., \& Salden, R. J. C. M. (2009). Theworkedexample effect: Not an artefact of lousy control conditions. Computers in HumanBehavior, 25, 258-266.

Shih, B., Koedinger, K. R., \& Scheines, R. (2008). A response time model for bottom-out hints as worked examples. In: Proceedings of the 1st International Conference on Educational Data Mining, Montreal, Canada.

Sweller, J. (1988). Cognitive load during problem solving: Effects on learning. Cognitive Science, 12, 257285.

Sweller, J. (2010). Element interactivity and intrinsic, extraneous and germane cognitive load. Educational Psychology Review, 22, 123-138.

Sweller, J., \& Cooper, G. A. (1985). The use of worked examples as a substitute for problem solving in learning algebra. Cognition and Instruction, 2, 59-89.

Sweller, J., van Merriënboer, J. J. G., \& Paas, F. (1998). Cognitive architecture and instructional design. Educational Psychology Review, 10, 251-296.

Trafton, J. G., \& Reiser, B. J. (1993). The contributions of studying examples and solving problems to skill acquisition. In M. Polson (Ed.), Proceedings of the Fifteenth Annual Conference of the Cognitive Science Society. Hillsdale, NJ: Erlbaum.

VanLehn, K., Lynch, C., Schulze, K., Shapiro, J. A., Shelby, A., Taylor, D., et al. (2005). The Andes physics tutoring project: Five years of evaluations. International Journal of Artificial Intelligence in Education, $15,1-47$.

Van Merriënboer, J. J. G., \& Sweller, J. (2005). Cognitive load theory and complex learning: Recent developments and future direction. Educational Psychology Review, 17, 147-178.

Van Merriënboer, J. J. G., Schuurman, J. G., de Croock, M. B. M., \& Paas, F. (2002). Redirecting learners' attention during training: Effects on cognitive load, transfer test performance and training efficiency. Learning and Instruction, 12, 11-37.

Ward, M., \& Sweller, J. (1990). Structuring effective worked examples. Cognition and Instruction, 7, 1-39.

Watanabe, A., Kato, N., \& Kato, T. (2002). Effects of creatine on mental fatigue and cerebral hemoglobin oxygenation. Neuroscience Research, 42, 279-285. 